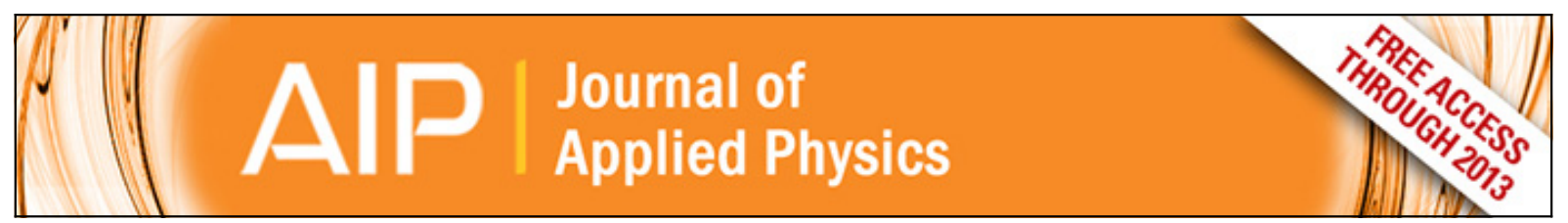

Effect of low-Raman window position on correlated photon-pair generation in a chalcogenide Ge11.5As24Se64.5 nanowire

J. He, C. Xiong, A. S. Clark, M. J. Collins, X. Gai, D.-Y. Choi, S. J. Madden, B. Luther-Davies, and B. J. Eggleton

Citation: Journal of Applied Physics 112, 123101 (2012); doi: 10.1063/1.4769740

View online: http://dx.doi.org/10.1063/1.4769740

View Table of Contents: http://scitation.aip.org/content/aip/journal/jap/112/12?ver=pdfcov

Published by the AIP Publishing

AlP Re-register for Table of Content Alerts 


\title{
Effect of low-Raman window position on correlated photon-pair generation in a chalcogenide $\mathrm{Ge}_{11.5} \mathrm{As}_{\mathbf{2 4}} \mathrm{Se}_{\mathbf{6 4 . 5}}$ nanowire
}

\author{
J. He, ${ }^{1}$ C. Xiong, ${ }^{1, a)}$ A. S. Clark, ${ }^{1}$ M. J. Collins, ${ }^{1}$ X. Gai, ${ }^{2}$ D.-Y. Choi, ${ }^{2}$ S. J. Madden, ${ }^{2}$ \\ B. Luther-Davies, ${ }^{2}$ and B. J. Eggleton ${ }^{1}$ \\ ${ }^{1}$ Centre for Ultrahigh-bandwidth Devices for Optical Systems (CUDOS), Institute of Photonics and Optical \\ Science (IPOS), School of Physics, University of Sydney, NSW 2006, Australia \\ ${ }^{2}$ CUDOS, Laser Physics Centre, Research School of Physics and Engineering, Australian National University, \\ Canberra ACT 0200, Australia
}

(Received 16 August 2012; accepted 12 November 2012; published online 18 December 2012)

\begin{abstract}
We investigated correlated photon-pair generation via spontaneous four-wave mixing in an integrated chalcogenide $\mathrm{Ge}_{11.5} \mathrm{As}_{24} \mathrm{Se}_{64.5}$ photonic nanowire. The coincidence to accidental ratio, a key measurement for the quality of correlated photon-pair sources, was measured to be only 0.4 when the photon pairs were generated at $1.9 \mathrm{THz}$ detuning from the pump frequency due to high spontaneous Raman noise in this regime. However, the existence of a characteristic low-Raman window at around $5.1 \mathrm{THz}$ in this material's Raman spectrum and dispersion engineering of the nanowire allowed us to generate photon pairs with a coincidence to accidental ratio of 4.5 , more than 10 times higher than the $1.9 \mathrm{THz}$ case. Through comparing the results with those achieved in chalcogenide $\mathrm{As}_{2} \mathrm{~S}_{3}$ waveguides which also exhibit a low Raman-window but at a larger detuning of $7.4 \mathrm{THz}$, we find that the position of the characteristic low-Raman window plays an important role on reducing spontaneous Raman noise because the phonon population is higher at smaller detuning. Therefore the ultimate solution for Raman noise reduction in $\mathrm{Ge}_{11.5} \mathrm{As}_{24} \mathrm{Se}_{64.5}$ is to generate photon pairs outside the Raman gain band at more than $10 \mathrm{THz}$ detuning. (C) 2012 American Institute of Physics. [http://dx.doi.org/10.1063/1.4769740]
\end{abstract}

\section{INTRODUCTION}

The implementation of integrated photonic quantum technologies, such as secure quantum communication ${ }^{1}$ and quantum computation, requires correlated photon-pair sources in the telecommunication band, which can be generated via spontaneous down conversion or spontaneous four-wave mixing (SFWM) in integrated photonic devices fabricated from highly nonlinear materials. Currently, the most commonly used integrated circuits are periodically poled $\chi^{(2)}$ waveguides, ${ }^{2,3}$ which require bulky and accurate temperature control to achieve quasi-phase matching, and silicon-oninsulator $\chi^{(3)}$ devices, ${ }^{4-7}$ which have poor performance at high power due to two-photon absorption (TPA) and freecarrier absorption (FCA).

Recently chalcogenide glasses including $\mathrm{As}_{2} \mathrm{~S}_{3}$ and $\mathrm{Ge}_{11.5} \mathrm{As}_{24} \mathrm{Se}_{64.5}$ (abbreviation $\mathrm{Ge}_{11}$ hereafter) based correlated photon-pair sources in the telecommunication band have attracted a lot of interest ${ }^{8-12}$ because of their high $\chi^{(3)}$ nonlinearity, negligible TPA and FCA, and compatibility with photonic integration. ${ }^{13}$ As amorphous materials, the chalcogenide glass platform does suffer from noise generated by spontaneous Raman scattering ( $\mathrm{SpRS}$ ), which impacts detrimentally the performance of a correlated photon-pair source. ${ }^{9}$ Various ideas including cooling a device ${ }^{10}$ and using the characteristic low-Raman window ${ }^{8,11}$ have been investigated thoroughly to reduce SpRS noise for the $\mathrm{As}_{2} \mathrm{~S}_{3}$ rib waveguide based photon-pair source. Nanowires fabricated from $\mathrm{Ge}_{11}$ are able to achieve more than 10 times

${ }^{a)}$ Electronic mail: chunle@physics.usyd.edu.au. higher nonlinearity than $\mathrm{As}_{2} \mathrm{~S}_{3}$ rib waveguides ${ }^{14}$ and therefore can be a more energy-efficient platform for correlated photon-pair generation. ${ }^{12}$ Reference 12 has also proposed that the SpRS noise can be mitigated if photon pairs can be generated in the low-Raman regime. However there is no experimental demonstration reported yet.

In this paper, we experimentally demonstrate the generation of correlated photon pairs in a chalcogenide $\mathrm{Ge}_{11}$ nanowire. We show that similar to $\mathrm{As}_{2} \mathrm{~S}_{3}, \mathrm{Ge}_{11}$ also exhibits a low-Raman window enabling us to generate correlated photon pairs at room temperature with a CAR of 4.5, ten times higher than the case without Raman suppression. The high nonlinearity allowed us to operate at a power level that is one order of magnitude lower than in $\mathrm{As}_{2} \mathrm{~S}_{3}$. However, as the low-Raman window of $\mathrm{Ge}_{11}$ is positioned only $5.1 \mathrm{THz}$ below the pump, less than the 7.4 THz detuning for $\mathrm{As}_{2} \mathrm{~S}_{3}$, the phonon population at this lower detuning is higher and thus causes the Raman suppression in $\mathrm{Ge}_{11}$ to be less effective than in $\mathrm{As}_{2} \mathrm{~S}_{3}$. This implies that the pairs should be generated at a larger detuning of $>10 \mathrm{THz}$ where more sophisticated dispersion engineering becomes necessary. ${ }^{12}$

\section{EXPERIMENT}

\section{A. Principle of operation and device}

Similar to previous experiments in $\chi^{(3)}$ nonlinear devices, we used SFWM to generate correlated photon pairs. As illustrated in Fig. 1(a), two pump photons at $\omega_{\mathrm{p}}$ are annihilated to create a signal photon at higher frequency $\omega_{\mathrm{s}}=\omega_{\mathrm{p}}$ $+2 \pi \nu$ and an idler photon at lower frequency $\omega_{\mathrm{i}}=\omega_{\mathrm{p}}-2 \pi \nu$ simultaneously in the same polarization mode as the pump. 


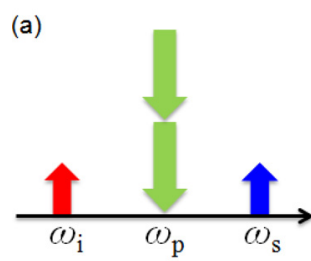

(b)

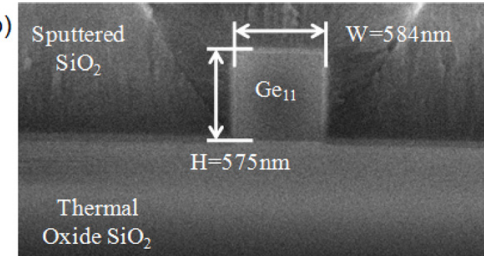

FIG. 1. (a) Schematic of the SFWM process. (b) A scanning electron microscope (SEM) image of the cross-section of the $\mathrm{Ge}_{11}$ nanowire.

Building upon our initial theoretical work, ${ }^{8}$ the SFWM pair generation rate in the pulsed pump regime can be estimated as

$$
r_{\mathrm{SFWM}}(v)=\sigma \Delta v\left(\gamma P_{0} L\right)^{2} \operatorname{sinc}^{2}\left(\frac{\beta_{2}(2 \pi v)^{2}}{2} L+\gamma P_{0} L\right)
$$

where $\sigma$ is the product of pulse width and laser repetition rate, $\Delta v$ is the characteristic filter bandwidth for the photon pair with a frequency detuning of $v$ from pump, $\gamma$ is the nonlinear coefficient, $P_{0}$ is the coupled peak power in the waveguide, $L$ is the waveguide length (propagation loss is ignored for simplifying the model), and $\beta_{2}$ is the dispersion coefficient at the pump wavelength. The sinc function in Eq. (1) incorporates the phasematching condition and determines the SFWM bandwidth.

During the generation of photon pairs via SFWM in an amorphous glass platform, SpRS also occurs and reduces the correlation between the photon pairs. The SpRS generation rate is estimated as ${ }^{15}$

$$
r_{\mathrm{SpRS}}(v, T)=C_{\text {coupling }} \sigma \Delta v P_{0} \operatorname{Lg}(v) N(v, T) \frac{1}{v},
$$

which includes a coupling coefficient $C_{\text {coupling, the phonon }}$ density of states $g(v)$, temperature $T$, and boson population $N(v, T)=n(v, T)+1$ for Stokes and $N(v, T)=n(v, T)$ for anti-Stokes with

$$
n(v, T)=\frac{1}{\mathrm{e}^{\frac{h v}{k T}}-1},
$$

where $h$ is Planck's constant and $k_{\mathrm{B}}$ is the Boltzmann constant.

The $\mathrm{Ge}_{11}$ waveguide used in the experiment was a $7 \mathrm{~mm}$ long, $584 \mathrm{~nm}$ wide, and $575 \mathrm{~nm}$ high nanowire, deposited on a silica-on-silicon substrate with a sputtered $\mathrm{SiO}_{2}$ cladding and is shown in Fig. 1(b). The nanowire supports both TE and TM modes with similar dispersion profile, but we will focus on the TM mode because it had a lower propagation loss, which was measured to be $1.7 \mathrm{~dB} \cdot \mathrm{cm}^{-1}$. The nonlinear coefficient $\gamma$ was evaluated to be $130 \mathrm{~W}^{-1} \mathrm{~m}^{-1}$, and the dispersion was calculated to be approximately $155 \mathrm{ps} \cdot \mathrm{nm}^{-1} \mathrm{~km}^{-1}$ across a wavelength range of 1530 $1550 \mathrm{~nm}^{14}$

Using Eqs. (1)-(3), we plot the normalized photon generation rate for $\gamma P_{0} L=0.1$ via SFWM and SpRS as a function of pump-idler frequency detuning $v$, shown in Fig. 2. It can be seen that the SpRS spectrum of the $\mathrm{Ge}_{11}$ nanowire

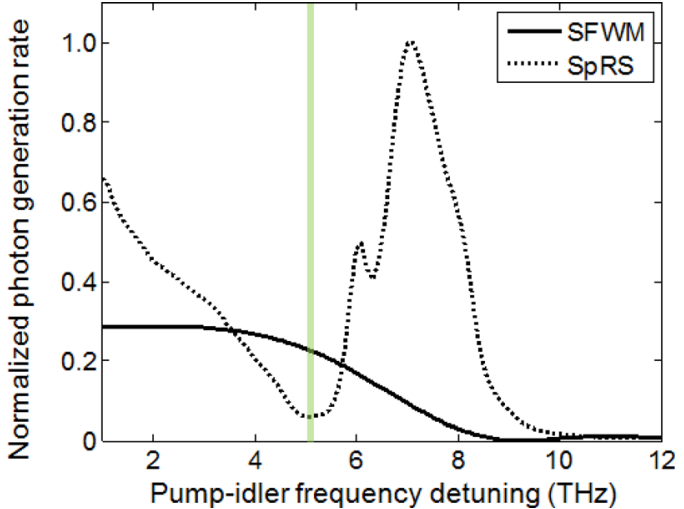

FIG. 2. Calculation of the photon generation rates versus detuning from the pump for $\gamma P_{0} L=0.1$. The green bar indicates the low-Raman window.

exhibits a characteristic low-Raman window at $v=5.1 \mathrm{THz}$ which can be reached by the SFWM bandwidth, as indicated by the green bar. The key here is to generate photon pairs in this window and demonstrate a reduced Raman noise.

\section{B. Experimental setup}

Figure 3 shows the experimental setup. A mode-locked pulsed tunable fiber laser (Pritel) produced 10 ps pulses at a repetition rate of $10 \mathrm{MHz}$. These pulses passed through an isolator, followed by a $980 / 1550 \mathrm{~nm}$ wavelength division multiplexer (WDM) to block any residual cavity pump photons. An attenuator and a polarization controller conditioned the pulses before reaching a band-pass filter (BPF) centered at the pump wavelength, positioned directly before the chip to block any SpRS photons generated in the preceding silica fibers. The pump pulses were then coupled into the waveguide using a lensed fiber. Once the photon pairs were generated, they were collected at the output of the waveguide using another lensed fiber and then post-selected by a wavelength division device and band-pass filters (BPFs) before they were fed into InGaAs/InP single-photon detectors (SPDs, id-Quantique, id201). The combination of the wavelength division device and filters gave an out-of-band noise suppression of about $100 \mathrm{~dB}$.

Both SPDs were operated at the gated mode and were triggered at an effective rate of $5 \mathrm{MHz}$ (the maximum trigger

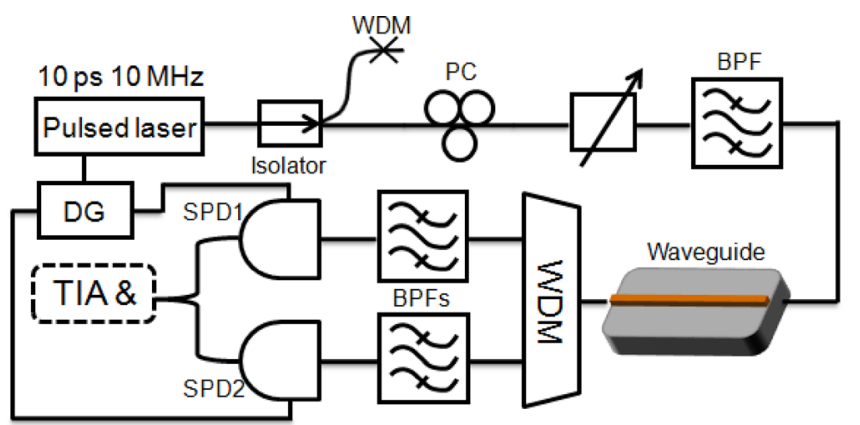

FIG. 3. Experimental setup. PC: polarization controller; BPF: band-pass filter; WDM: wavelength division multiplexer; SPD: single-photon detector; DG: delay generator; TIA: time interval analyzer. For different SFWM detuning frequency, different optical components were used to post-select different signal and idler wavelengths. 
frequency of our detection system) using alternate radio frequency pulses from the laser synchronized with the optical pulses. A delay generator (DG) was used to synchronize the detector gating and the optical pulses. The detector gate width for both SPDs was set at $5 \mathrm{~ns}$, and the detection probability of both SPDs was set at $10 \%$ to reduce dark counts to 9 $\times 10^{-5}$ and $6 \times 10^{-5}$ per gate, respectively. The dead time was set at $10 \mu$ s to avoid significant after-pulsing probability. The detection signals from the two SPDs were sent to a time interval analyzer (TIA) to record the coincidence events. The simultaneous detection of a photon at both detectors was recorded as a coincidence $C_{\text {raw }}$. We measured the accidental coincidence counts $A$ by counting the detection events separated by an additional trigger period, where no photon-pair correlation exists. Then, the true coincidence count can be determined by $C=C_{\text {raw }}-A$. We define the coincidence to accidental ratio $(\mathrm{CAR})$ as $\mathrm{CAR}=C / A$. A higher $\mathrm{CAR}$ suggests a better signal to noise ratio of the quantum system.

\section{Experimental results and analysis}

Figure 2 shows that photon pairs can be generated over a broad bandwidth. For comparison, we performed photonpair generation at both far detuning at $5.1 \mathrm{THz}$ and near detuning at $1.9 \mathrm{THz}$. To post-select the pairs generated in the low-Raman window $(v=5.1 \mathrm{THz})$, we tuned the pump wavelength to $1530.1 \mathrm{~nm}$ and employed a coarse WDM, which consisted of 8 channels centered at 1471, 1491, 1511, $1531,1551,1571,1591$, and $1611 \mathrm{~nm}$, respectively, with a $13 \mathrm{~nm}$ channel bandwidth, to suppress pump photons and separate signal and idler photons at 1491.3 and $1571.0 \mathrm{~nm}$. Whilst for the pairs generated at $1.9 \mathrm{THz}$ detuning, we pumped at $1545.4 \mathrm{~nm}$ and used a standard arrayed waveguide grating (AWG) to post-select the pair photons. The AWG output channels were spaced evenly by $100 \mathrm{GHz}$ and had a bandwidth of $55 \mathrm{GHz}$. After the separation of signal and idler photons, tunable band-pass filters with $1 \mathrm{~nm}$ bandwidth were used to further suppress out-of-band noise in both far and near detuning experiments. Taking into account coupling efficiencies, all component losses and detector efficiencies, the overall photon collection efficiencies are estimated to be $0.26 \%$ and $0.17 \%$ at $5.1 \mathrm{THz}$ detuning and $0.05 \%$ and $0.04 \%$ at $1.9 \mathrm{THz}$ detuning, both for signal and idler channels, respectively.

At first we examined the single count rate dependence on coupled peak power and the results are plotted in Fig. 4. A clear quadratic dependence was obtained at far detuning whilst the trend was linear at near detuning. According to Eqs. (1) and (2), quadratic dependence is a signature of SFWM, which means that the SFWM process dominates at far detuning because the noise is reduced in the low-Raman window. However at near detuning the SpRS process dominates leading to the linear dependence. It should be noted that the single count rate for near detuning should be higher than that for far detuning because, according to Fig. 2, the SFWM photon-pair generation rate is about the same, but the SpRS photon generation rate is much higher at near detuning. The count rates were similar in our measurement for both signal and idler due to the much lower photon

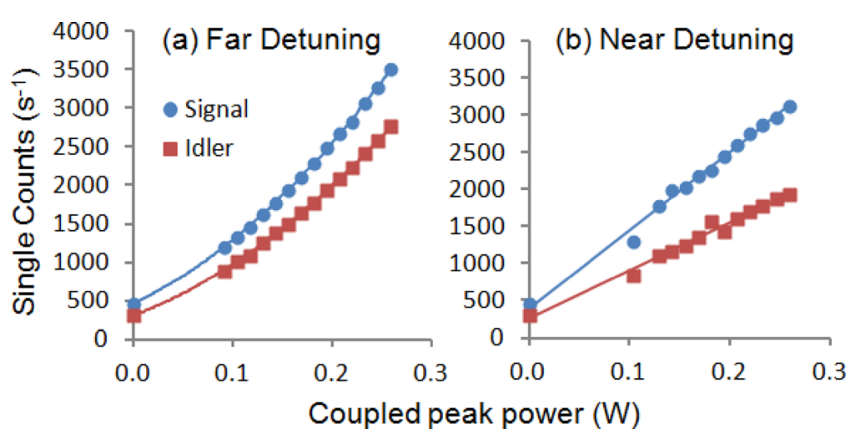

FIG. 4. Measured single count rate as a function of coupled peak power for (a) far and (b) near detuning. Blue circles are for signal and red squares are for idler. Solid lines are quadratic fits in (a) and linear fits in (b).

collection efficiencies introduced by the AWG used in the near detuning experiment. The imbalance between the count rates for the signal and idler photons observed in both cases is due to the different dark counts in the two SPDs, different photon collection efficiencies, and different Stokes and antiStokes SpRS photon rates in the signal and idler channels.

To show the enhancement of CAR, we measured the coincidence count rate at different pump powers and the results are plotted in Fig. 5 for both far and near detuning. Coincidences (blue triangles) were consistently higher than accidentals (red squares), which confirmed the generation of correlated photon pairs. The true coincidence count rate is quadratically dependent on pump power for both cases because true coincidences can only come from SFWM. However, for pairs generated at $v=1.9 \mathrm{THz}$, true coincidences (green circles) were lower than accidentals, and for pairs generated at $v=5.1 \mathrm{THz}$, true coincidences were significantly higher than accidentals due to much less SpRS noise. As we mentioned above, the true coincidence should be the same for two cases, but it was lower at near detuning because of the extra loss from the AWG.

We then plot CAR, the ratio of true coincidences and accidentals, as the function of average number of generated pairs per pulse $\mu$ in Fig. 6 for both detuning cases. From Fig. 6 we notice that firstly the maximum CAR was about 4.5 for far detuning and was 10 times higher than that for near detuning. Second, the CAR increased initially with $\mu$ in both cases because of the competition between real photon

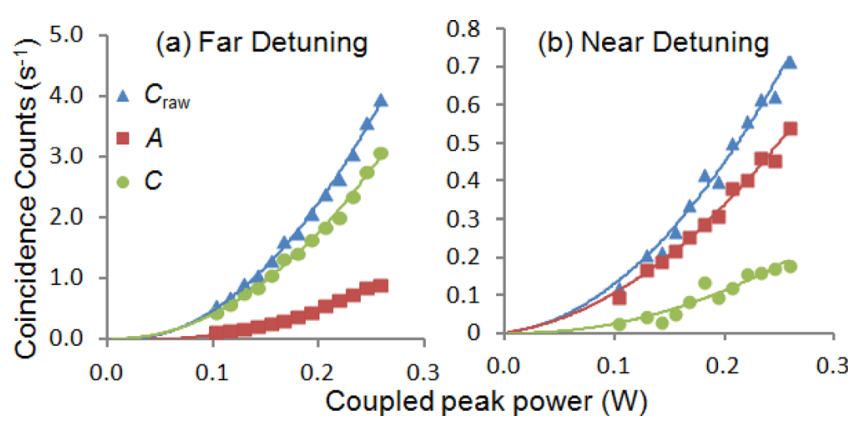

FIG. 5. Measured coincidence count rate as a function of coupled peak power for (a) far and (b) near detuning. Blue triangles are for raw coincidences $C_{\text {raw }}$, green circles for true coincidences $C$ and red squares for accidentals $A$. Solid lines are quadratic fits. 


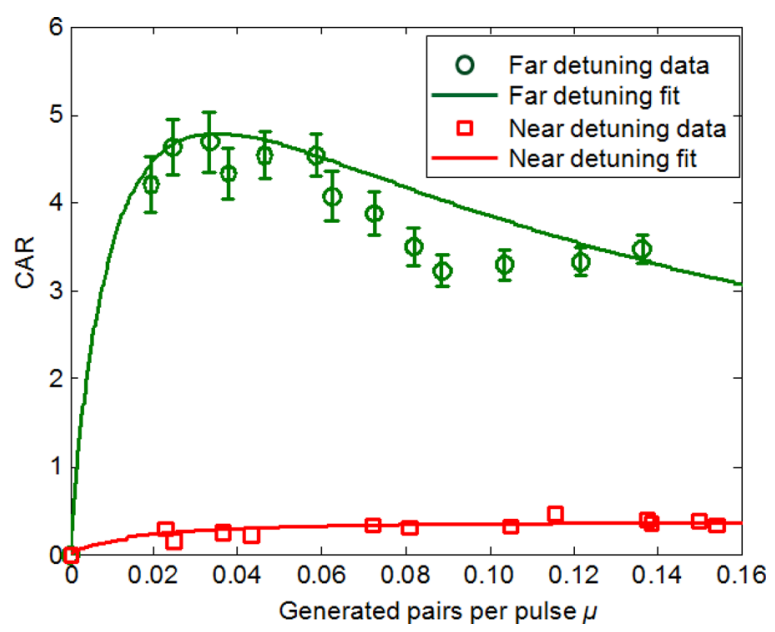

FIG. 6. CAR as the function of average number of generated pairs per pulse $\mu$ for far (green circles) and near detuning (red squares). Poissonian error bars are used for far detuning data, and error bars are too small for near detuning data to be shown in the plot. Solid lines are fits using Eq. (4).

counts and dark counts and then remained fairly constant for near detuning in the range investigated, whilst remaining constant at low $\mu$ and dropping at higher $\mu$ for far detuning. The CAR for near detuning did not drop as expected with increased $\mu$ because the dominant noise was SpRS rather than multiple-pair generation. This behavior was similar to the observation in Ref. 5. The CAR plot for far detuning does not show an obvious peak as the CAR normally does in a SFWM based photon-pair generation system free from SpRS noise, ${ }^{6}$ which means there still remains a moderate amount of Raman noise even in the low-Raman window.

To quantitatively understand the impact of the characteristic low-Raman window on CAR, we use a simple model to fit the CAR plots. If the overall photon collection efficiencies are $\eta_{\mathrm{s}}$ and $\eta_{\mathrm{i}}$, the probability of generating a noise photon per pulse is $\mu_{\mathrm{s}}$ and $\mu_{\mathrm{i}}$, and the detector dark count probability per triggering is $d_{\mathrm{s}}$ and $d_{\mathrm{i}}$, all for the signal and idler arms respectively, the formula for CAR is ${ }^{6}$

$$
\mathrm{CAR}=\frac{\eta_{\mathrm{s}} \eta_{\mathrm{i}} \mu}{\left[\eta_{\mathrm{s}}\left(\mu+\mu_{\mathrm{s}}\right)+d_{\mathrm{s}}\right]\left[\eta_{\mathrm{i}}\left(\mu+\mu_{\mathrm{i}}\right)+d_{\mathrm{i}}\right]} .
$$

In Eq. (4), $\mu, \eta_{\mathrm{s}, \mathrm{i}}$, and $d_{\mathrm{s}, \mathrm{i}}$ are all known quantities from the experiment. Using Eq. (4) we can fit the CAR plots in Fig. 6 for both near and far detuning cases to find the free parameters $\mu_{\mathrm{s}}$ and $\mu_{\mathrm{i}}$. In doing so we find that the ratios of $\mu_{\mathrm{s}}$ and $\mu_{\mathrm{i}}$ in near and far detuning cases show that SpRS is reduced in both the signal and idler channels by a factor of 11.8 and 6.9, respectively. On the other hand, using Eq. (2) we calculate the ratios of SpRS at two detuning cases to be 13.1 and 7.6 for the signal and idler channels, respectively, in good agreement with the values extracted from the CAR plots fit. Therefore we confirm that the 10 times enhancement on CAR is from the suppression of SpRS noise at far detuning.

Finally we compare the results achieved in $\mathrm{Ge}_{11}$ with those reported in a chalcogenide $\mathrm{As}_{2} \mathrm{~S}_{3}$ waveguide using a similar technique to suppress SpRS noise. ${ }^{11}$ The maximum CAR of 4.5 in $\mathrm{Ge}_{11}$ is much lower than 16.8 reported in Ref. 11. One obvious reason is that the dark count of the id 201 detector module used here is much higher than that of the id210 module used in Ref. 11. If we apply the dark count parameter of the id210 to Eq. (4) whilst we keep all other parameters the same, the CAR will be about 12 at the same pair generation rate. A useful photon-pair source for quantum communication requires a CAR over 10 to obtain a $<5 \%$ quantum bit error rate. ${ }^{16}$ This means that our $\mathrm{Ge}_{11}$ photon-pair source will be immediately useful to quantum applications by changing to low-noise detectors. The other important reason is that in the far detuning experiment for the $\mathrm{Ge}_{11}$ platform, the SpRS noise was suppressed to some extent, but the suppression was not as efficient as for the $\mathrm{As}_{2} \mathrm{~S}_{3}$ platform. To understand the difference, we have to notice that the characteristic low-Raman window of $\mathrm{Ge}_{11}$ is at $5.1 \mathrm{THz}$ detuning, smaller than the $7.4 \mathrm{THz}$ detuning for $\mathrm{As}_{2} \mathrm{~S}_{3}$ because the molecular bond of $\mathrm{Ge}_{11}$ is formed from heavier atoms and vibrates slower than that of $\mathrm{As}_{2} \mathrm{~S}_{3}$. Using Eq. (2) we evaluate the SpRS photon generation rate at the low-Raman window for $\mathrm{Ge}_{11}$ and $\mathrm{As}_{2} \mathrm{~S}_{3}$ and find that the value in $\mathrm{Ge}_{11}$ is twice of that in $\mathrm{As}_{2} \mathrm{~S}_{3}$ because the characteristic low-Raman window of $\mathrm{Ge}_{11}$ is located at a lower frequency detuning where the phonon population is higher. This means that the ultimate solution of getting rid of SpRS noise is to generate pairs at a larger detuning of $>10 \mathrm{THz}$ where SFWM phase matching can be achieved by careful dispersion engineering using the high-accuracy atomic layer deposition techonology. ${ }^{12}$ On the other hand, comparing the power used in this experiment to that in Ref. 11, we find that the $\mathrm{Ge}_{11}$ platform required 10 times less power because of its higher nonlinearity.

\section{CONCLUSION}

In conclusion, SpRS noise is the major source of noise for the chalcogenide $\mathrm{Ge}_{11}$ glass based correlated photon-pair source. We have demonstrated that the SpRS noise can be partially mitigated by generating the photon pairs in the lowRaman window of this material. By comparing the performance with that of $\mathrm{As}_{2} \mathrm{~S}_{3}$, we confirm that the $\mathrm{Ge}_{11}$ platform is more energy-efficient but infer that careful dispersion engineering is required to extend the SFWM bandwidth to over $10 \mathrm{THz}$ to further suppress SpRS noise.

\section{ACKNOWLEDGMENTS}

This work was supported by the Centre of Excellence, Federation Fellowship (FF0776056), Discovery Early Career Researcher Award (DECRA, DE120100226) and Future Fellowship (FT110100853) programs of the Australian Research Council (ARC). The Centre for Ultrahighbandwidth Devices for Optical Systems (CUDOS) is an ARC Centre of Excellence (Project No. CE110001018).

\footnotetext{
${ }^{1}$ N. Gisin and R. Thew, Nat. Photonics 1, 165 (2007).

${ }^{2}$ J. Chen, A. J. Pearlman, A. Ling, J. Fan, and A. Migdall, Opt. Express 17, 6727 (2009).

${ }^{3}$ M. Hunault, H. Takesue, O. Tadanaga, Y. Nishida, and M. Asobe, Opt. Lett. 35, 1239 (2010).

${ }^{4}$ J. E. Sharping, K. F. Lee, M. A. Foster, A. C. Turner, B. S. Schmidt, M. Lipson, A. L. Gaeta, and P. Kumar, Opt. Express 14, 12388 (2006).
} 
${ }^{5}$ S. Clemmen, A. Perret, S. K. Selvaraja, W. Bogaerts, D. van Thourhout, R. Baets, P. Emplit, and S. Massar, Opt. Lett. 35, 3483 (2010).

${ }^{6} \mathrm{~K}$. Harada, H. Takesue, H. Fukuda, T. Tsuchizawa, T. Watanabe, K. Yamada, Y. Tokura, and S. Itabashi, IEEE J. Sel. Top. Quantum Electron. 16, 325 (2010).

C. Xiong, C. Monat, A. S. Clark, C. Grillet, G. D. Marshall, M. J. Steel, J. Li, L. O'Faolain, T. F. Krauss, J. G. Rarity, and B. J. Eggleton, Opt. Lett. 36, 3413 (2011).

${ }^{8}$ C. Xiong, L. G. Helt, A. C. Judge, G. D. Marshall, M. J. Steel, J. E. Sipe, and B. J. Eggleton, Opt. Express 18, 16206 (2010).

${ }^{9}$ C. Xiong, G. Marshall, A. Peruzzo, M. Lobino, A. Clark, D.-Y. Choi, S. Madden, V. Zwiller, M. Thompson, J. Rarity, M. Steel, B. LutherDavies, B. Eggleton, and J. O'Brien, Appl. Phys. Lett. 98, 051101 (2011).
${ }^{10}$ A. S. Clark, M. J. Collins, A. C. Judge, E. C. Mägi, C. Xiong, and B. J. Eggleton, Opt. Express 20, 16807 (2012).

${ }^{11}$ M. J. Collins, A. S. Clark, J. He, D.-Y. Choi, R. J. Williams, A. C. Judge, S. J. Madden, M. J. Withford, M. J. Steel, B. Luther-Davies, C. Xiong, and B. J. Eggleton, Opt. Lett. 37, 3393 (2012).

${ }^{12}$ X. Gai, R. P. Wang, C. Xiong, M. J. Steel, B. J. Eggleton, and B. LutherDavies, Opt. Express 20, 776 (2012).

${ }^{13}$ B. Eggleton, B. Luther-Davies, and K. Richardson, Nat. Photonics 5, 141 (2011).

${ }^{14}$ X. Gai, D.-Y. Choi, S. J. Madden, and B. Luther-Davies, Opt. Express 20, 13513 (2012)

${ }^{15}$ R. J. Kobliska and S. A. Solin, Phys. Rev. B 8, 756 (1973).

${ }^{16} \mathrm{H}$. Takesue and K. Inoue, Opt. Express 13, 7832 (2005). 